\title{
Traffic control and intelligent vehicle highway systems: A survey*
}

\author{
L.D. Baskar, B. De Schutter, J. Hellendoorn, and Z. Papp
}

If you want to cite this report, please use the following reference instead:

L.D. Baskar, B. De Schutter, J. Hellendoorn, and Z. Papp, "Traffic control and intelligent vehicle highway systems: A survey," IET Intelligent Transport Systems, vol. 5, no. 1, pp. 38-52, Mar. 2011.

Delft Center for Systems and Control

Delft University of Technology

Mekelweg 2, 2628 CD Delft

The Netherlands

phone: +31-15-278.24.73 (secretary)

URL: https: //www.dcsc.tudelft.nl

*This report can also be downloaded via https://pub. deschutter.info/abs/09_043.html 


\title{
Traffic Control and Intelligent Vehicle Highway Systems: A Survey
}

\author{
L. D. Baskar*, B. De Schutter*, J. Hellendoorn*, Z. Papp ${ }^{\dagger}$ \\ * Delft Center for Systems and Control, Delft University of Technology \\ Mekelweg 2, 2628 CD Delft, The Netherlands \\ email: $\{1 . d$. baskar, j.hellendoorn $\}$ etudelft.nl, b@deschutter.info \\ $\dagger$ TNO Science and Industry \\ P.O.Box 155, 2600 AD Delft, The Netherlands \\ email: zoltan.pappetno.nl
}

\begin{abstract}
Traffic congestion in highway networks is one of the main issues to be addressed by today's traffic management schemes. Automation combined with the increasing market penetration of on-line communication, navigation, and advanced driver assistance systems will ultimately result in intelligent vehicle highway systems (IVHS) that distribute intelligence between roadside infrastructure and vehicles and that — in particular on the longer term — are one of the most promising solutions to the traffic congestion problem. In this paper, we present a survey on traffic management and control frameworks for IVHS. First, we give a short overview of the main currently used traffic control methods for freeways. Next, we discuss IVHS-based traffic control measures. Then, various traffic management architectures for IVHS such as PATH, Dolphin, Auto21 CDS, etc. are discussed and a comparison of the various frameworks is presented. Finally, we sketch how existing traffic control methodologies could fit in an IVHS-based traffic control set-up.
\end{abstract}

\section{Introduction}

Due to the ever-increasing traffic demand, modern societies with well-planned road management systems, and sufficient infrastructures for transportation still face the problem of traffic congestion. This results in loss of travel time, and huge societal and economic costs. Constructing new roads could be one of the solutions for handling the traffic congestion problem, but it is often less feasible due to political and environmental concerns. An alternative would be to make more efficient use of the existing infrastructure. In this paper, we will consider the latter approach with a special focus on freeway traffic management and control.

Traffic management and control approaches are used to control the traffic flows and to prevent or reduce traffic jams, or more generally to improve the performance of the traffic system. Possible performance measures in this context are throughput, travel times, safety, fuel consumption, emissions, reliability, etc. Currently implemented traffic management approaches primarily make use of roadside-based traffic control measures (such as ramp metering, traffic signals, dynamic route information panels, and dynamic speed limits) and infrastructure-based equipment (including sensors and traffic control centres). These measures and the corresponding equipment will be indicated by the term "roadside infrastructure" in the remainder of the paper. 
As an extension to the current traffic control approaches, advanced technologies in the field of communication, control, and information systems have been combined with the existing transportation infrastructure and equipment [53]. This marks the emergence of a next level/next generation of traffic control and management approaches and serves as the motivation for "Intelligent Transportation Systems" (ITS) or "Intelligent Vehicle Highway Systems" (IVHS) [31,95]. ITS/IVHS incorporate intelligence in both the roadway infrastructure and in the vehicles with the intention of reducing congestion and environmental impact, and of improving traffic performance, by exploiting the distributed nature of the system and by making use of cooperation and coordination between the various vehicles and the various elements of the roadside infrastructure. In the remainder of this paper, we will use IVHS as a generic word to indicate these systems.

IVHS comprise traffic management systems, driver information systems, and vehicle control systems. In particular, these vehicle control systems are aimed at developing an automated vehiclehighway system that shifts the driver tasks from the driver to the vehicle [105]. These driver tasks include activities such as steering, braking, and making control decisions about speeds and safe headways. Automated Highway Systems (AHS) go one step further than IVHS and involve complete automation of the driving task. For better (network-wide) coordination of traffic activities, AHS also distribute the intelligence between the vehicles and the roadside infrastructure. In this paper, we will focus on AHS and on the relations and interactions between the vehicles in the AHS and the roadside infrastructure. In particular, we will consider the control aspects of these systems.

An important component of IVHS and AHS are the intelligent vehicles (IVs), which sense the environment around them using sensors (such as radar, lidar, or machine vision techniques) and strive to achieve more efficient vehicle operation either by assisting the driver (via advisories or warnings) or by taking complete control of the vehicle (i.e., partial or full automation) [11]. These IVs also support vehicle-vehicle and vehicle-roadside communication.

Based on the extent to which the roadside and vehicle could work together (without human driver intervention), we can discern different types of AHS as follows:

- Autonomous vehicle systems: Vehicles are equipped with sensors and computers to operate without roadside infrastructure assistance and without coordination with neighbouring vehicles.

- Cooperative vehicle systems: Vehicles use sensors and wireless communication techniques to coordinate their manoeuvres with neighbouring vehicles without any roadside intervention.

- Infrastructure-supported systems: Vehicles communicate with each other and guidelines for decision making purposes are provided by the roadside infrastructure.

- Infrastructure-managed systems: Vehicles indicate their desired actions such as lane changes, exits, and entries to the roadside infrastructure. The roadside system then provides the instructions for inter-vehicle coordination of these manoeuvres.

- Infrastructure-controlled systems: The roadside infrastructure takes entire control of the vehicle operations, monitors the traffic, and optimises the vehicle operations in such a way that the network is utilised as well as possible.

Each of these categories is characterised by its own time scale and the "region" covered, i.e., the number of other vehicles and traffic control measures involved in the process. In this context autonomous and cooperative systems typically operate in the milliseconds to seconds range and cover small groups of neighbouring vehicles. On the other hand, the infrastructure-based systems (i.e., managed and controlled) typically work in the seconds to hours range and involve a much larger number of vehicles and several freeway links or even entire freeway networks. 
The objectives of this paper are twofold. The first objective is to provide a survey of traffic control frameworks for IVHS that integrate the intelligence of both the roadside infrastructure and the IVs to improve the traffic performance. The second objective is to discuss the potential application of control design methods that are currently used for traffic control purposes to IVHS-based traffic management and control systems. More specifically, this paper is organised as follows. Section 2 presents an overview of the current control methods that are applied for traffic control and management. In Section 3 we give an overview of intelligent vehicles and IV-based control measures. In Section 4 we present existing IVHS frameworks that combine roadside infrastructure and vehicles for efficient traffic management, and in Section 5 we provide a comparative analysis of these frameworks. Finally, Section 6 briefly sketches how the currently used control design methods presented in Section 2 could potentially be applied in these IV-based traffic control frameworks. This section also presents topics for future work.

\section{Control design methods}

In the literature different control methodologies have been presented for controlling and managing a traffic network in which vehicles are driven by humans [27,54,76]. In this section, we will discuss the control design methodologies for freeway traffic control that are currently most often used in practice such as

- Static feedback control,

- Optimal control and model predictive control,

- Artificial intelligence (AI) techniques.

Throughout this section, we will consider ramp metering as a typical application for freeway traffic control and use it to illustrate the different control methods on a common example. Note, however, that the methods can also be used for other traffic control measures such as variable speed limits, lane closures, shoulder lane openings, etc.

\section{Ramp metering}

Ramp metering $[59,74,98]$ is a traffic control measure that can be applied at the on-ramps of freeways and that is basically implemented via traffic signals located at the freeway entrances. The purpose of a ramp metering installation is to regulate the flow of traffic that is allowed to enter the freeway from the on-ramp such that the freeway capacity is utilised efficiently.

For example, let us consider a freeway section with a mainstream origin and an on-ramp origin. The on-ramp origin $o$ is receiving a traffic demand $d_{o}(\mathrm{veh} / \mathrm{h})$. Let $q_{o}(\mathrm{veh} / \mathrm{h})$ be the flow admitted by the traffic signal, let $q_{\text {cap }}$ be the freeway capacity (veh/h), and let $q_{\text {in }}$ be the upstream freeway flow (veh/h). When traffic congestion occurs, the maximum outflow from the traffic jam $q_{\text {con }}(\mathrm{veh} / \mathrm{h})$ is usually less than when compared to free-flow traffic $q_{\text {cap. }}$. This phenomenon is called capacity drop $[36,68]$ and should be prevented whenever possible. If no ramp metering is applied, then there is a possibility for congestion on the freeway due to the on-ramp vehicles in case the demand $d_{o}$ and the upstream flow $q_{\text {in }}$ are high. Using ramp metering, breakdown of the flow can be avoided and the outflow can be kept at a high level due to prevention of capacity drop, which in its turn can reduce the amount of time spent by the vehicles in the network. Obviously, applying ramp metering could result 


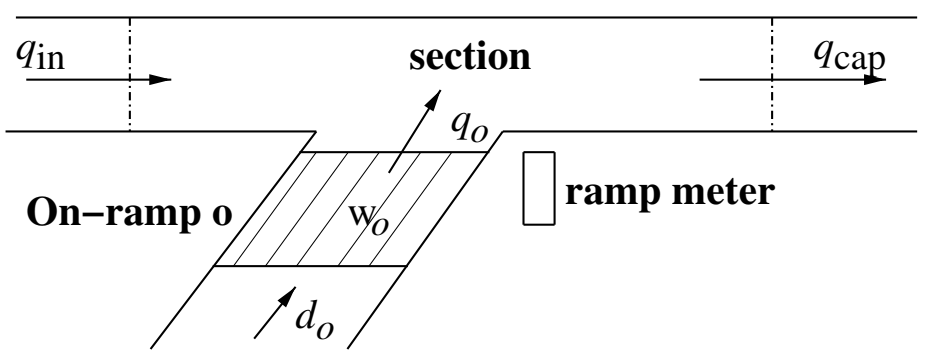

Figure 1: Ramp metering

in a queue with queue length $w_{o}$ on the on-ramp as shown in Figure 1. Hence, a trade-off has to be maintained between reducing the congestion on the freeway and keeping the on-ramp queues short.

The control methods we discuss below are operating in discrete time. This means that at each sample time instant $t=k T$ where $T$ is the sampling interval and the integer $k$ is the discrete-time sample step, measurements of the traffic are performed and fed to the traffic controller. The controller then uses this information to determine the control signal to be applied during the next sampling interval.

\subsection{Static feedback control}

\subsubsection{General concepts}

Dynamical systems can be controlled in two ways: using open-loop control and using closed-loop control. In an open-loop system, the control input does not depend on the output of the system, whereas in a closed-loop system, the control action is a function of the output of the system. Feedback or closed-loop control systems are suited for applications that involve uncertainties or modelling errors.

In "static" " feedback control methods, the controller gets measurements from the system and determines control actions based on the current state of the system in such a way that the performance of the system is improved. The main examples of static feedback controllers are state feedback controllers (where the feedback gain can be computed using, e.g., pole placement) and PID controllers (for which several tuning rules exist, such as the Ziegler-Nichols rules) [4]. However, the static feedback strategy in general does not handle any external constraints. This is a major drawback of this control scheme.

\subsubsection{Control application for conventional traffic}

A ramp metering system based on static feedback derives control actions (the metering rates) using real-time traffic measurements collected from the vicinity of the ramp meter. The two main local ramp metering strategies that use static feedback are $[78,94]$ :

- The demand-capacity strategy,

- ALINEA.

\footnotetext{
${ }^{1}$ By "static" we mean here that the control parameters of the feedback controller are taken to be fixed.
} 


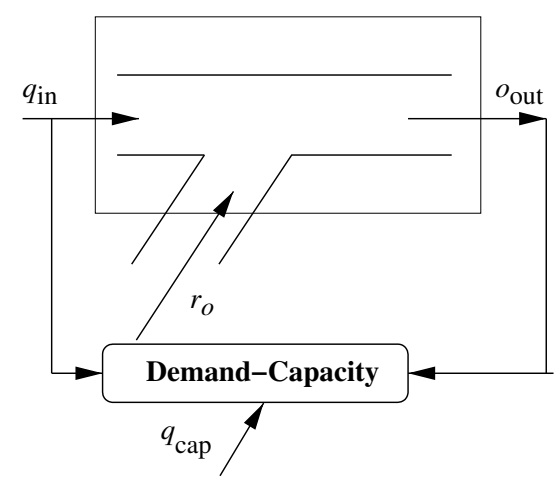

(a) Demand-capacity strategy

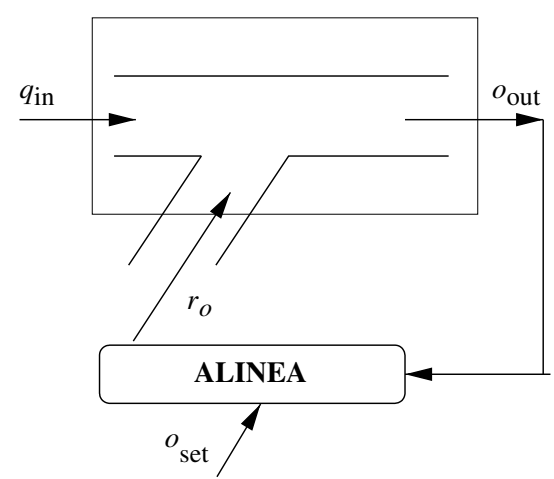

(b) ALINEA

Figure 2: Demand-capacity strategy and ALINEA.

The demand-capacity strategy can be formulated as follows (see also Figure 2(a)):

$$
r_{o}(k)= \begin{cases}q_{\mathrm{cap}}-q_{\mathrm{in}}(k-1) & \text { if } o_{\text {out }}(k) \leq o_{\mathrm{cr}} \\ q_{\mathrm{min}} & \text { otherwise }\end{cases}
$$

where $r_{o}(k)$ is the flow rate on the on-ramp at sample step $k, o_{\text {out }}(k)$ is the freeway occupancy downstream of the on-ramp, $o_{\mathrm{cr}}$ is the critical occupancy at which the traffic flow tends to be at its maximum, and $q_{\min }$ is the prespecified minimum ramp flow value. This strategy determines the flow rate value as the difference between the downstream capacity and the upstream flow, until the critical occupancy is reached. Once this critical occupancy is reached, the on-ramp flow rate is reduced to a minimum value to prevent the occurrence of congestion. Also this demand strategy attempts to solve or to prevent the congestion problem only after the freeway is operating at its maximum usage.

One of the best known ramp metering strategies is ALINEA [78] (or one of its extensions such as the METALINE algorithm [77]). The basic ALINEA strategy is a closed-loop algorithm that determines the ramp metering rate in such a way that the downstream occupancy from the on-ramp is kept at a desired or prespecified value $o_{\text {set }}$ (see also Figure 2(b)):

$$
r_{o}(k)=r_{o}(k-1)+K_{\mathrm{r}}\left(o_{\text {set }}-o_{\text {out }}(k)\right)
$$

where $r_{o}(k)$ is the flow rate on the ramp at sample step $k, o_{\text {out }}(k)$ is the freeway occupancy downstream of the ramp, and $K_{\mathrm{r}}$ is a regulator parameter. One of the difficulties of this method is the determination of appropriate set-points.

Although the static feedback control strategies discussed above are based on real-time measurements, they are implemented at a local level only, based on the information that is obtained locally (i.e., in the vicinity of an on-ramp).

\subsection{Optimal control and model predictive control}

Now we discuss two dynamic control methods that apply optimisation algorithms to determine optimal control actions based on real-time measurements: optimal control and model predictive control. 


\subsubsection{General concepts}

\section{General concepts of optimal control}

Optimal control determines a sequence of admissible control actions that optimise a performance function by considering future demands and by satisfying the constraints $[20,57,96]$. A general discrete-time optimal control problem contains the following elements:

1. Dynamical system model equations,

2. An initial state $\mathbf{x}_{0}$,

3. An initial time $t_{0}$,

4. Constraints,

5. Measurements,

6. A performance index $J$.

More specifically, consider a multi-input multi-output dynamical system expressed by the following equation:

$$
\mathbf{x}(k+1)=\mathbf{f}(\mathbf{x}(k), \mathbf{u}(k), \mathbf{d}(k))
$$

where $\mathbf{x} \in \mathbb{R}^{n}$ is an $n$-vector of states, $\mathbf{u} \in \mathbb{R}^{m}$ is an $m$-vector of manipulatable control inputs, $\mathbf{f}$ is a continuously differentiable function, and $\mathbf{d}$ is the disturbance vector.

For a given time horizon $K$, the optimal control problem consists in determining a sequence of control vectors $\mathbf{u}(0), \mathbf{u}(1), \ldots, \mathbf{u}(K-1)$ in such a way that the performance index $J$ takes on the minimum possible value subject to the initial conditions, system dynamics, and constraints, i.e.,

Minimise

$$
J=\vartheta[\mathbf{x}(K)]+\sum_{k=0}^{K-1} \varphi[\mathbf{x}(k), \mathbf{u}(k), \mathbf{d}(k)]
$$

subject to

$$
\begin{array}{ll}
\mathbf{x}(0)=\mathbf{x}_{0} & \\
\mathbf{x}(k+1)=\mathbf{f}(\mathbf{x}(k), \mathbf{u}(k), \mathbf{d}(k)) & \text { for } k=0, \ldots, K-1, \\
\mathbf{u}_{\min }(k) \leq \mathbf{u}(k) \leq \mathbf{u}_{\max }(k) & \text { for } k=0, \ldots, K-1, \\
\mathbf{c}(\mathbf{x}(k), \mathbf{u}(k), k) \leq \mathbf{0} & \text { for } k=0, \ldots, K-1,
\end{array}
$$

where $\vartheta$ and $\varphi$ are twice differentiable, nonlinear functions and are called the terminal cost and Lagrangian respectively, $\mathbf{u}_{\min }$ and $\mathbf{u}_{\max }$ are bounds for the control variables, $\mathbf{c}$ expresses path constraints imposed on the state $\mathbf{x}$ and the control trajectories $\mathbf{u}$ over the period $\left[t_{0}, t_{0}+K T\right]$, and $\mathbf{d}$ is assumed to be known over the period $\left[t_{0}, t_{0}+K T\right]$. Thus the performance criterion depends on the initial state, and the whole time history of the system's state and the control variables. There are two basic approaches to solve the above optimal control problem: calculus of variations $[34,45]$ and dynamic programming [10].

The main drawback of optimal control is that the method is essentially an open-loop control approach and thus suffers from disturbances and model mismatch errors. Next, we will discuss model predictive control, which uses feedback and a receding horizon approach to overcome some of the drawbacks optimal control. 


\section{General concepts of model predictive control}

Model Predictive Control (MPC) $[17,67,83]$ has originated in the process industry and it has already been successfully implemented in many industrial applications. MPC is a feedback control algorithm that can handle constrained, complex dynamical systems [21,23]. The main difference between optimal control and MPC is the rolling horizon approach used in MPC (this essentially means that the optimal control is performed repeatedly but over a limited horizon). On the one hand, this results in a suboptimal performance compared to optimal control (at least in the absence of disturbances). However, on the other hand, the rolling horizon approach introduces a feedback mechanism, which allows to reduce the effects of possible disturbances and of model mismatch errors.

The underlying concept of the MPC controller is based on on-line optimisation and uses an explicit prediction model to obtain the optimal values for the control measures subject to system dynamics and constraints. At each time step $k$, the MPC controller first measures or determines the current state $\mathbf{x}(k)$ of the system. Next, the controller uses (on-line) optimisation and an explicit prediction model to determine the optimal values for the control measures over a given prediction period determined by the prediction horizon $N_{\mathrm{p}}$ (see Figure 3 ). In order to reduce the computational complexity of the problem, one often introduces a constraint of the form $\mathbf{u}(k+j)=\mathbf{u}(k+j-1)$ for $j=N_{\mathrm{c}}, \ldots, N_{\mathrm{p}}-1$, where $N_{\mathrm{c}}$ is called the control horizon.

The optimal control inputs are then applied to the system in a receding horizon approach as follows. At each control step $k$ only the first control sample $\mathbf{u}^{*}(k)$ of the optimal control sequence $\mathbf{u}^{*}(k), \ldots, \mathbf{u}^{*}\left(k+N_{\mathrm{c}}-1\right)$ is applied to the system. Next, the prediction horizon is shifted one step forward, and the prediction and optimisation procedure over the shifted horizon is repeated using new system measurements.

MPC for linear systems subject to a quadratic objective function and linear constraints can be solved using quadratic programming. Other types of MPC problems in general require global or multi-start local optimisation methods such as sequential quadratic programming, pattern search, or simulated annealing [79].

Just as optimal control MPC can take into account constraints on the inputs and outputs, and it can also deal with multi-input multi-output systems. MPC has an advantage over optimal control due to receding horizon approach. This feedback mechanism of MPC makes the controlled system more robust to uncertainties and disturbances. Nevertheless, MPC still has some of the drawbacks of optimal control such as computational complexity, the need of an explicit model for prediction purposes, and the fact that the external inputs and disturbances need to be known fairly accurately in advance for the entire prediction horizon.

\subsubsection{Control application for conventional traffic}

Optimal control can be applied to ramp metering as follows [60-62]. A second-order macroscopic METANET model can be used to model a freeway traffic system. The METANET model represents a network as a directed graph with links (corresponding to freeway stretches with uniform characteristics) and vertices (corresponding to on-ramps, intersections, etc.). In METANET each link is divided into segments. The state vector $\mathbf{x}$ for the control problem consists of densities $\rho_{m, i}$, and mean speeds $v_{m, i}$ of every segment $i$ of every link $m$, and queue lengths $w_{o}$ at every origin $o$ of the network. The control vector $\mathbf{u}$ consists of the ramp metering rates $r_{o}$ of every on-ramp $o$ under control. The process disturbance vector $\mathbf{d}$ contains demands $d_{o}$ at the origin links. All these values are assumed to be known over the entire simulation period. When controlling traffic, the controller needs to have a goal or objective to achieve, such as minimising the total time spent in a traffic network, minimis- 

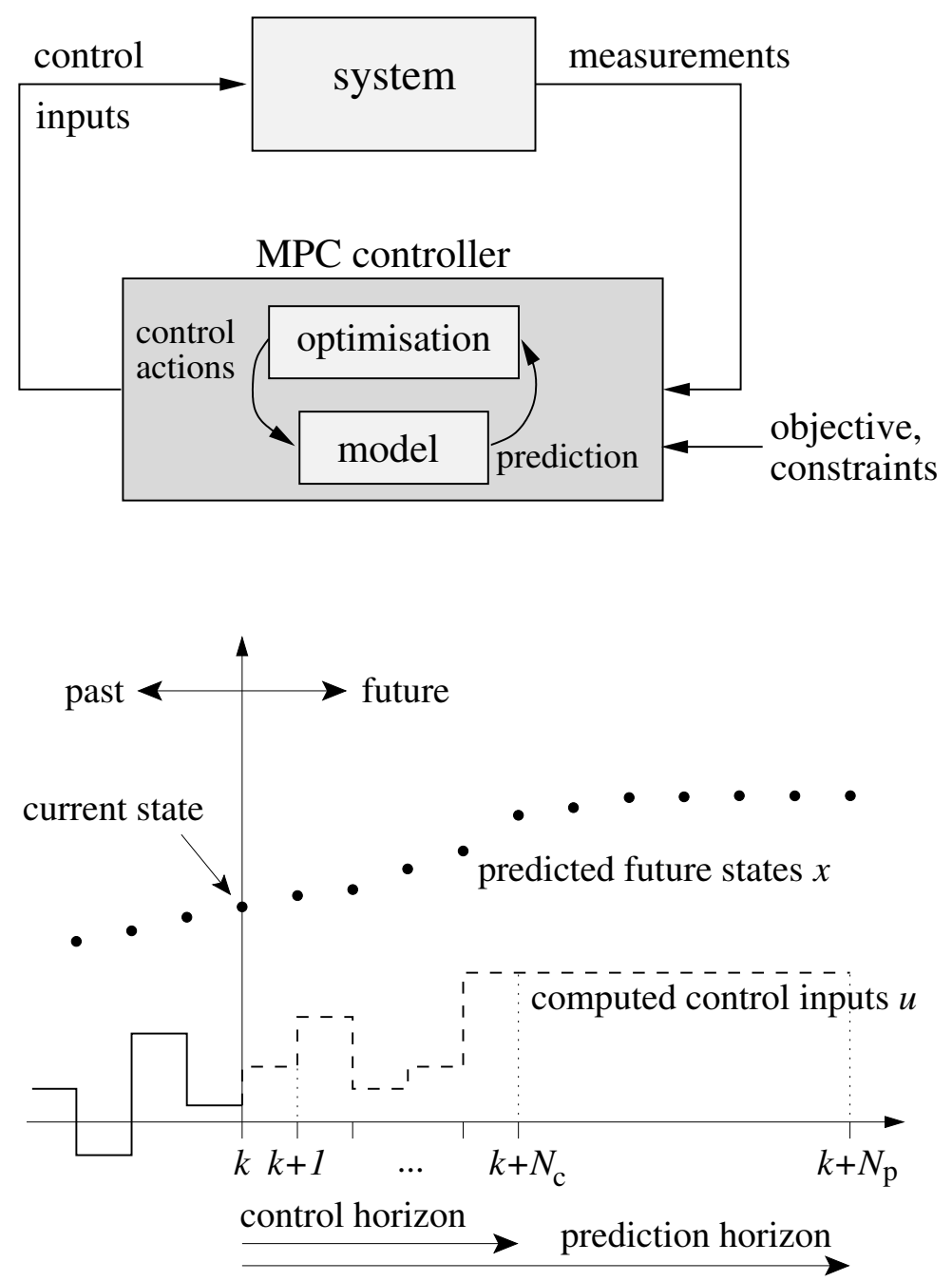

Figure 3: Model predictive control 
ing the total distance travelled, minimising the total fuel consumption, minimising the queue lengths, maximising the safety of vehicles in the network, etc., or a combination of these objectives.

Using the optimal control algorithm, the controller computes the optimal control values, based on the assumed demands $d_{o}$. In reality, these demands cannot be known in advance. However, often the future demand can be estimated reasonably well from upstream and downstream measurements in combination with historical data.

MPC for ramp metering and for conventional road-side based non-IV traffic management has been developed and implemented in $[9,42,43]$. Similar models as used for optimal control approach are considered. The optimisation problem can be solved in the same way as for optimal control. The main differences are that in MPC a shorter horizon is selected, that a control horizon is used - such that the control signals (in this case, the metering rates $r_{o}$ of every on-ramp $o$ under control) are allowed to vary till the control horizon, after which the rates are taken to remain constant, - and that the optimal control inputs are applied in a moving horizon scheme. The latter feature results in a more robust operation of the traffic control system.

\subsection{Artificial intelligence techniques}

\subsubsection{General concepts}

Artificial Intelligence (AI) techniques aim at imitating aspects of human intelligence and thinking while solving a problem by introducing human intelligence (to perceive a situation, to reason about it, and to act accordingly) into computer programs [22]. AI techniques are mainly used in decision support systems, and one way to classify them is as follows [73,86,97, 109]:

- Case-based reasoning,

- Fuzzy logic,

- Rule-based systems,

- Artificial neural networks,

- Multi-agent systems.

Case-based reasoning, as the name suggests, solves a problem using the knowledge that was gained from previously experienced similar situations (cases) [1,86]. In this way, this technique learns the way a new problem is solved, tests the proposed solution using simulation methods, and stores the new solution in a database. A disadvantage of this approach is that it might not be clear what should be done for a case that is not yet present in the case base. However, new cases could be added on-line to deal with this problem.

Fuzzy logic systems, like humans, can handle situations where the available information about the system is vague or imprecise $[58,73]$. To deal with such situations, fuzzy sets are used to qualify the variables of the system in a non-quantitative way. Fuzzy sets are characterised using membership functions (e.g. Gaussian, triangle, or normal) that take a value between 0 and 1 , and that indicate to what degree a given element belongs to the set (e.g., a speed could be $60 \%$ "high" and $40 \%$ "medium"). The membership degrees can then be used to combine various rules and to derive conclusions. This process consists of three parts: fuzzification, inference, and defuzzification. Fuzzification involves the transformation of a value of a variable into a fuzzy value, by linking it a given fuzzy set and determining a value for degree of membership. Inference uses a set of rules based on expert 
opinions and system knowledge and combines them using fuzzy set operators such as complement, intersection, and union of sets. Defuzzification converts the fuzzy output of the inference step in to a crisp value using techniques such as maximum, mean-of-maxima, and centroid defuzzification. One of main difficulties of a fuzzy system can be the selection of appropriate membership functions for the input and output variables. Moreover, fuzzy systems are often combined with other AI techniques for their complete deployment.

Rule-based systems solve a problem using "if-then" rules [40,89]. These rules are constructed using expert knowledge and stored in an inference engine. The inference engine has an internal memory that stores rules and information about the problem, a pattern matcher, and a rule applier. The pattern matcher searches through the memory to decide which rules are suitable for the problem, and next the rule applier chooses the rule to apply. These systems are suited to solve problems where experts can make confident decisions. However, this system works only with already created rules and in its basic implementation it does not involve learning.

Artificial neural networks try to mimic the way in which the human brain processes information $[39,111]$. These systems are useful in solving nonlinear problems where the rules or the algorithm to find solutions are difficult to derive. The basic processing unit of a neural network is called neuron or node. Each node fires a new signal when it receives a sufficiently high input signal from the other connected nodes. These nodes are organised in layers (an input layer, an output layer, and a number of hidden layers) and are interconnected by links or synapses, each associated with weights. A disadvantage is that artificial neural networks are non-informative models, and do not provide an explanation for the outcomes or for any failure that may occur in the process.

An agent is an entity that can perceive its environment through sensors and act upon its environment through actuators in a such way that the performance criteria are met [52]. Multi-agent systems consist of a network of agents that are interacting among themselves to achieve specified goals. A high-level agent communication language is used by the agents for communication and negotiation purposes. Multi-agent systems can be applied to model complex systems, but their dynamic nature and the interactions between agents may give rise to conflicting goals or resource allocation problems.

\subsubsection{Control application for conventional traffic}

Of the AI methods discussed above case-based reasoning and fuzzy logic are most often described in literature for traffic control purposes. In practice, rule-based systems are also used very often for traffic management and control.

Many decision support systems have been proposed for traffic management purposes such as FRED (Freeway Real-Time Expert System Demonstration) [87, 112], the Santa Monica Smart Corridor Demonstration Project [55,88], and TRYS [25, 63, 72].

In $[25,44,47,112]$ case-based systems are developed for a broad range of scenarios that may occur in a traffic network. This initial case-based system is in principle generated using historical data or off-line simulations. In $[44,47]$ each scenario in the case base is evaluated over a prediction horizon using simulation and can be characterised by "inputs" such as the current traffic state, the traffic control measure applied (e.g., ramp metering, lane closures, etc.), or expected incidents (duration of incidents), and by "outputs" such as predicted average values of the traffic states or predicted values for performance measures. Hence, the case-based system maintains an input-output relation for each considered scenario. Once the initial case base is constructed, the traffic control centre can use this case-based system as the basis for assessing a particular traffic problem and for determining the most appropriate control scenarios. In case the current situation has not been addressed before, a new, real-life scenario of the traffic system can be evaluated and added to the case-based system along 
Table 1: Comparison of control design methods

\begin{tabular}{|c|c|c|c|c|c|}
\hline Control method & $\begin{array}{c}\text { Computational } \\
\text { complexity }\end{array}$ & $\begin{array}{l}\text { Constraints } \\
\text { (hard) }\end{array}$ & $\begin{array}{l}\text { Future } \\
\text { inputs }\end{array}$ & Model-based & Scalability \\
\hline Static feedback & low & no & no & not explicitly & localised \\
\hline $\begin{array}{l}\text { Optimal control } \\
\text { and MPC }\end{array}$ & high & yes & yes & model-based & system-wide \\
\hline AI-based & medium & no & no & not explicitly & localised \\
\hline
\end{tabular}

with effect of the control measures. Hence, learning and updating the case-based system with newly encountered cases serves as the main advantage and motivation for the application of this system in the traffic control field.

Although this technique has been useful for controlling small networks, adding each possible case for a large-scale traffic network can be a difficult task. To solve this problem and to handle large-scale traffic networks, a possible solution has been proposed in [30]. The solution of [30] considers a largescale network as small subnetworks and uses fuzzy logic to combine different cases in the case base. By using fuzzy logic, a precise match between the considered actual case and the relevant cases in the case-based system will not be required.

As indicated before fuzzy systems can be used when accurate information of the traffic model is difficult to obtain or is not available [15,63]. A fuzzy logic controller for ramp metering with a description of the various steps (fuzzification, inference, and defuzzification) is presented in [107]. Several fuzzy sets that can relate a variable (input, output) to a particular situation can be defined such as fuzzy sets for local speed, local traffic flow, queue occupancy, metering rate and local occupancy. Using fuzzification input variables such as speeds, flows, occupancy levels in the vicinity of the fuzzy ramp meter controller, and output variables such as metering rates can be translated to fit the defined fuzzy sets and to obtain values for the degree of membership. Next, these values are fed to the inference engine, which is constructed using a set of rules based on the experience of traffic control centre operators and on off-line simulations. These rules are to be kept as simple and easy to understand and to modify. The result of the inference is then transformed into a crisp value in the defuzzification step, after which the final result is applied to the traffic system or presented to the operator of the traffic control centre for further assessment.

Related work is described in $[44,70]$.

\subsection{Comparison}

We will now compare the control design methods discussed above based on the following directions:

- Computational complexity,

- Inclusion of hard constraints,

- Inclusion of future inputs,

- Model-based or not,

- Scalability.

The results of this analysis are shown in Table 1. 


\section{Computational complexity}

Although the control design methods discussed above provide suboptimal performance (except for optimal control in the error-free case), the computational complexity of these methods does vary depending on their application area. Static feedback control methods have low on-line computational requirements, but they are in practice mainly applicable to small-scale systems. The on-line computational time required for AI techniques is somewhat higher but still much less than that of optimal control and MPC since the latter use on-line optimisation. All three control techniques also require off-line computations for model identification and model parameter calibration as well as for parameter tuning, training, or rule generation. These off-line computational requirements are mainly determined by the model and its complexity (model order, degree of nonlinearity, etc.).

\section{Inclusion of hard constraints}

Consideration of hard constraints is an inevitable aspect to be included when controlling and managing traffic flows. Hard constraints are those that are to be necessarily satisfied. In general, static feedback control does not consider the external constraints. This lack of inclusion of constraints also proves to be one of the major drawbacks of the static feedback methods. Most AI methods also do not explicitly take (hard) constraints into account. Optimal control and MPC on the other hand use optimisation and as a consequence they can explicitly include hard constraints when determining the optimal control actions.

\section{Inclusion of future inputs}

Static feedback controllers do not consider the external future inputs. Since optimal control and MPC are prediction-based, they can also take into account knowledge about future inputs (e.g., in traffic control one might detect traffic flows using detectors located upstream and use this information). In general, AI techniques may also use predictions.

\section{Model-based or not}

All the control approaches explicitly or implicitly need a model to collect data and/or for tuning. However, by model-based we mean in particular whether the control method considers an explicit model in order to determine the optimal control actions. Although the parameters of a static feedback controller may be tuned using model-based simulations, the controller does not explicitly include a model of the traffic system inside it. In a similar way the rules or cases of an AI-based controller may also be determined using simulation models, but the models are not explicitly present in the controller. On the other hand, optimal controllers and MPC controllers in general use an explicit model of the (traffic) system as a prediction model when determining the optimal control actions.

\section{Scalability}

Based on the control structure used, we can categorise control and management systems $[3,32,93]$ as follows:

- Centralised,

- Distributed, 
- Decentralised,

- Hierarchical.

In a centralised set-up all control measures are managed from one single point, the traffic control centre. However, in practice such an approach is not feasible due to the lack of scalability and robustness, the communication requirements, and the computational complexity. One way to deal with these issues is to use a distributed approach in which several local controllers each control their own region or set of measures and in which the local controllers communicate and coordinate their actions. If only communication is present but there is no coordination, we have a decentralised control structure, which is usually easier to implement but also less optimal. Hierarchical control frameworks combine features of centralised and distributed control: they use a hierarchical framework with several control layers in which lower-level controllers take care of the fast dynamics in a small region of influence, and higher-level controllers take care of the slower dynamics and the coordination over a larger region of influence.

In this context, static feedback controllers are mostly localised and are used to control smallscale systems (in a central way) or as lower-level controllers in a hierarchical control framework. Most AI-based control methods (except for multi-agent approaches) are also mostly localised and can also be used in a hierarchical control framework. MPC (and optimal control) determines the control actions based on the current and predicted future states of the system and thus allows for a systemwide coordination of the control actions. In principle, MPC can be used at all levels of a hierarchical control framework but due to its computational complexity it is less suited for the lower levels. In addition, recently some distributed MPC methods have been developed [18].

In this section we have presented a brief overview of applied traffic control methods that use currently existing traffic control measures. In the next sections we will consider future traffic control systems based on intelligent vehicles and automated highway systems.

\section{Intelligent vehicles and traffic control}

\subsection{Intelligent vehicles}

We can divide IV application areas into three categories depending on the level of support provided to the driver [13]:

- Advisory systems use a human machine interface (HMI) (e.g., optic or acoustic) to provide an advisory or warning to the drivers. Some examples include blind spot warning, parking assistance, lane departure warning systems, and drowsy driver monitoring.

- Semi-autonomous systems can take partial control of vehicle manoeuvres and often use haptic (meaning "based on the sense of touch") measures to assist the driver and can take partial control of vehicle manoeuvres. Examples are an intelligent speed adaptation system using an active accelerator pedal that exerts a counterforce at speeds over the speed limit, or a forward collision mitigation or avoidance system that first warns the driver via seat vibrations and next starts to brake in case the driver does not react to the warning.

- Fully autonomous systems take complete control of vehicle operations and eliminate the driver from the control loop. Examples include fully automated adaptive cruise control and anti-lock braking systems. 
IV-based control measures can be further classified based on their manoeuvres [12]:

- Lateral sensing and control systems assist drivers in controlling the lateral movements of the vehicle. Applications of such systems are lane departure warning systems, lane change assist systems, and parallel parking assist systems.

- Longitudinal sensing and control systems mainly help in controlling forward and backward movements of the vehicle, and maintaining safe speeds and safe inter-vehicle distances. Typical applications are adaptive cruise control, forward collision warning systems, and intelligent speed adaptation.

- Integrated lateral and longitudinal sensing and control systems provide both lateral and longitudinal support to the driver. Such applications can combine the functionalities of adaptive cruise control and lane keeping assistance systems to assist the driver in performing manoeuvres.

\subsection{IV-based traffic management}

The currently implemented traffic control and management systems are mainly using intelligence in the roadside infrastructure for controlling and managing the traffic system, However, such a system does not make use of the significant benefits offered by the intelligence - including the additional control, sensing, and communication capabilities - provided by IVs. So one way to improve performance and safety of the current transportation systems is by applying automation and advanced intelligent control methods to roadside infrastructure and vehicles. Based on the way a traffic control and management approach utilises the automated vehicles, we can categorise the ongoing research on automated driving for traffic management as follows:

- Automated platooning,

- Autonomous IVs.

We will now briefly explain these two approaches.

An interesting functionality that is allowed by full automation is to arrange the vehicles in closely spaced groups called "platoons" [16]. In a platoon, the first vehicle is called "leader" and the remaining vehicles are called "followers". To avoid collisions, intra-platoon spacing (i.e., vehicle spacing within a platoon) is kept very small and the inter-platoon spacing is kept larger $[65,105]$. In the literature, many control frameworks, mainly intended to study inter-vehicle communication technologies and to control the platoon manoeuvres in cooperation with the roadside infrastructure, have been developed and investigated $[5,41,50,84,102]$. Also, frameworks that allow communication between the (partially or fully) automated individual vehicles and with the nearby roadside infrastructure have been proposed [26].

The other main category of IV-based traffic management approaches involves individual autonomous IVs and is mainly based on self-organisation and distributed intelligence among the automated vehicles with no control from the roadside infrastructure. In this autonomous driving system, the vehicles try to aggregate themselves in groups, make their decisions based on local information only, and perform manoeuvres in a cooperative fashion. Modelling and analysis of such group behaviour mechanisms in traffic management is an active area of research $[33,64,66,80]$.

In this paper, we focus on the first approach, as this approach most probably allows for a smoother transition from the current, exclusively roadside-based traffic control system towards a mixed IVroadside traffic management system in which the intelligence between the roadside infrastructure and the intelligent vehicles are integrated so as to improve the traffic performance. 


\subsection{IV-based control measures}

In this section, we discuss various IV technologies that support both roadside traffic control measures and automated platoons of IVs. The main functional areas of IV systems that support traffic management and control on freeways are:

- Adaptive Cruise Control (ACC),

- Intelligent Speed Adaptation (ISA),

- Dynamic route guidance.

\subsubsection{Adaptive cruise control}

Many vehicles have already been deployed with partial automation of the driving task by the use of the standard conventional cruise control [85].

An Adaptive Cruise Control (ACC) system is a radar-based system that extends the conventional cruise control system and that is designed to sense the vehicle immediately in front on the same lane, and to automatically adjust the speed of the equipped vehicle to that of the preceding vehicle so as to maintain a safe inter-vehicle distance [28,29]. If there is no predecessor, then ACC retains the preset speed that was selected by driver.

Variations of ACC are available in the market, among which high-speed ACC and stop-and-go ACC are popular [81]. High-speed ACC uses a radar to measure speed and distance of the vehicle ahead so as to maintain a safe distance between the vehicles. This ACC system is designed to operate at medium (above $40 \mathrm{~km} / \mathrm{h}$ ) to higher speeds, because at this range of speeds, a sensor can discriminate between non-moving targets and moving vehicles. A radar can sense a low relative velocity with respect to a moving predecessor and a high relative velocity with respect to stationary objects. However, high-speed ACC systems have difficulties to operate at low speeds. Stop-and-go ACC is designed to work with low speeds $(0 \mathrm{~km} / \mathrm{h}$ to $40 \mathrm{~km} / \mathrm{h})$ that prevail on urban roads. Basically, the stop-and-go ACC acts as a distance controller rather than a speed controller.

Although ACC vehicles are able to handle many traffic situations reliably [75], there exist some adverse traffic conditions such as short-headway cut-ins, or sudden and strong deceleration of the upfront vehicle that prevent the ACC from sensing and reacting to the situation immediately. Cooperative ACC is a further enhancement of ACC systems that utilises existing communication technologies (e.g., ad hoc wireless networks) to obtain real-time information about the speed, acceleration, braking, position, headway, and yaw rate of the preceding vehicle in order to maintain a safe but small headway, and to ensure smooth driving [104].

Using cooperative ACC, vehicles can travel at reduced headways. Hence, with reduced headways between vehicles, traffic flow can be improved.

\subsubsection{Intelligent speed adaptation}

A standard speed limiter is a system that restricts the speed of the vehicle when the driver tries to exceed the maximum allowed driving speed. When the speed limiter is incorporated with the intelligence to adjust the maximum driving speed to the speed limit specified by the roadside infrastructure or to the prevailing location-based legal speed limit, and to provide feedback to the driver when that speed limit is exceeded, then we get the technology called Intelligent Speed Adaptation (ISA) [14,24]. 
ISA systems can be characterised based on the extent to which the driver is made aware of the situation when the speed limit is exceeded. This results in advisory, voluntary, and mandatory systems $[19,99]$.

Another characterisation for ISA systems can be made based on the speed limit itself as a fixed or a dynamic speed limit. In the fixed case, the driver is informed about the speed limit, which could be obtained from a static database. A dynamic speed limit system takes current road conditions such as bad weather, slippery roads, or major incidents into account before prescribing the speed limit.

ISA can influence the traffic flow by limiting the maximum speed of the vehicles depending upon the actual traffic flow conditions. As such congestion can be delayed or alleviated by delaying vehicles so that by the time they arrive at a congested region the congestion has diminished or has already been dissolved.

\subsubsection{Dynamic route guidance}

Nowadays, many individual vehicles are equipped with a route guidance or navigation system. Based on current or expected travel times, and delays due to congestion, a route guidance system advises a driver about the "best" route he can take to reach his requested destination $[69,91,108]$. This route recommendation mainly depends on the vehicle's current location. Using a GPS, the position of the vehicle can be determined accurately. This information can be looked up on a digital road map and be used to determine the possible routes to reach the destination. These route recommendations may be calculated within the equipped vehicle or communicated to the vehicle from the local traffic centre.

A possible categorisation of route guidance systems is static versus dynamic route guidance. When the possible routes are computed based on the average traffic conditions (e.g, based on historical data or on the type of roads (such as urban corridor, highway, local road, etc.)), then this scheme is referred to as static route guidance system. If the current traffic conditions such as traffic jams, dynamic speed limits, and on-line predictions of travel times using real-time traffic data are taken into account while computing the route recommendations, then we have a dynamic route guidance system $[56,110]$.

Dynamic route guidance systems can improve the traffic flow, by providing route recommendations to the vehicles such that congestion is prevented from occurring or such that the effects of traffic jams are mitigated.

\section{Control frameworks and architectures for IVHS}

Now we discuss the most important control architectures that have been developed for linking the roadside infrastructure and automated platoons. In particular, we consider the PATH, Dolphin, Auto21 CDS, CVIS, SafeSpot, and PReVENT frameworks.

\subsection{PATH framework}

The PATH architecture $[16,48,92,105,106]$ mainly focuses on the coordination of both roadsidevehicle and inter-vehicle activities.

The PATH framework considers a traffic network with many interconnected highways on which the vehicles are organised in platoons. The highways in the traffic network are considered to be divided into links (about $5 \mathrm{~km}$ long). A link is subdivided into segments (about $1 \mathrm{~km}$ long) with at least one exit or one entrance. A vehicle in the PATH framework is either considered as a leader, a follower, or a free agent (i.e., a one-vehicle platoon). 


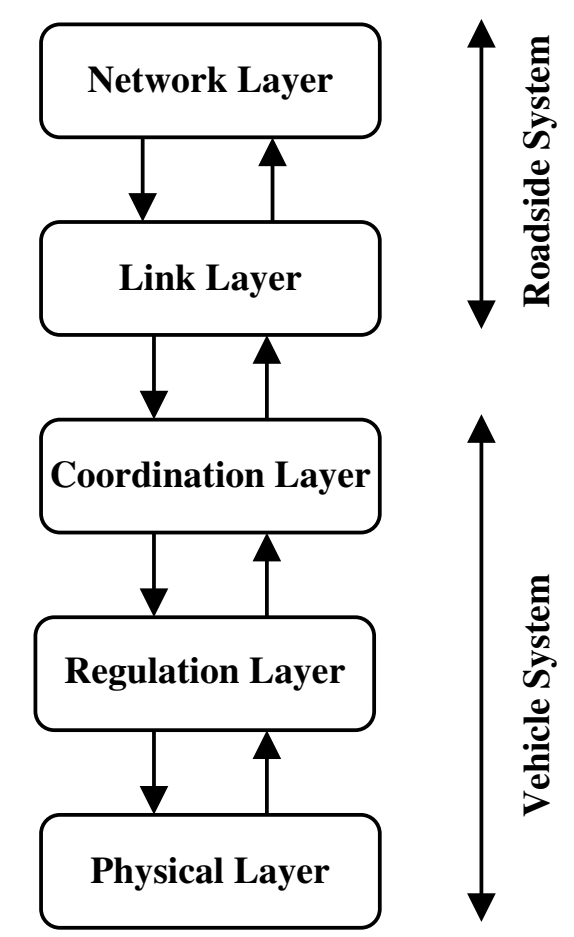

Figure 4: PATH architecture

The PATH framework is a hierarchical structure in which the control of the automated highway system is distributed into five functional layers as shown in Figure 4:

- Physical layer,

- Regulation layer,

- Coordination layer,

- Link layer,

- Network layer.

The lower levels in this hierarchy deal with faster time scales (typically in the milliseconds range for the physical layer up to the seconds range for the coordination layer), whereas for the higher-level layers the frequency of updating can range from few times per minute (for the link layer) to once every few minutes (for the network layer). The controllers in the physical, regulation, and coordination layer reside inside the vehicles. The physical and regulation controllers govern single vehicles, whereas the coordination layer involves several vehicles. The link layer and the network layer controllers are located at the roadside, with the link layer controllers managing single freeway segments, and the network layer controllers handling entire networks.

The same temporal and spatial division across different layers is also present in other frameworks that will be discussed in the next sections, such as the Dolphin framework and the Auto21 CDS framework.

Now we discuss each layer of the PATH framework in more detail, starting from the bottom of the hierarchy. 
The physical layer of each vehicle involves the actual dynamics of the vehicle. This layer has controllers that perform the actuation of the steering wheel, throttle, and brakes. It also contains the sensors in the vehicle that collect information about the speed, the acceleration, and the engine state of the vehicle, and send it to the regulation layer.

The regulation layer controller executes the tasks specified by the coordination layer (such lane changes, and splits or merges of platoons) by converting them into throttle, steering, and braking inputs for the actuators of the vehicle. The regulation layer controller within each vehicle uses feedback control laws to execute the lateral and longitudinal manoeuvres and also notifies the coordination controller in case of any failures or unsafe outcomes of the manoeuvres.

Next in the hierarchy is the coordination layer with a controller residing inside each vehicle. This layer receives the commands from the link layer (such as set-points or profiles for the speeds, or platoon sizes). A coordination layer controller allows coordination with other neighbouring platoons using messages or communication protocols, and checks which manoeuvres (like lane changes, splits, or merges) have to be performed by a vehicle in order to achieve the platoon size or path trajectory specified by the link controller.

The next layer in the control hierarchy is the link layer. This layer has a controller located at each link and is mainly responsible for path and congestion control. Each link controller receives commands from the network layer (such as routes for the platoons) and based on these commands, the link controller calculates the maximum platoon size, and the optimum platoon velocity for each segment in the link it is managing. The link controller also sets the local path (which lane to follow) for each platoon.

The top layer in the hierarchy is called the network layer. At this layer, the controller computes control actions that optimise the entire network. Its task is to assign a route for each vehicle or platoon that enters the highway ensuring that the capacity of each potential route is utilised properly. There will be one network layer controller for the considered traffic network.

The PATH framework has also been used in other projects such as, e.g., the AHSRA (Advanced Cruise-Assist Highway System Research Association) project launched by the Japanese Ministry of Land, Infrastructure and Transport [2].

\subsection{Dolphin framework}

The Japanese Dolphin framework developed in $[101,102]$ is similar to the PATH architecture. The name of this framework is inspired by the way in which groups of dolphins move and which is mimicked by the automated vehicles in the platoons. The Dolphin framework considers vehicles to be arranged as platoons and develops inter-vehicle communication technologies to carry out cooperative driving for the purpose of smooth merging and lane changing.

The Dolphin framework is composed of three layers as shown in Figure 5:

- Vehicle control layer,

- Vehicle management layer,

- Traffic control layer.

The vehicle controller within each vehicle senses the states and the conditions ahead of the vehicle such as vehicle speed and acceleration and sends this information to the vehicle management layer. The vehicle controller also receives commands for the vehicle's steering actions and determines the actions for the vehicle actuators. 


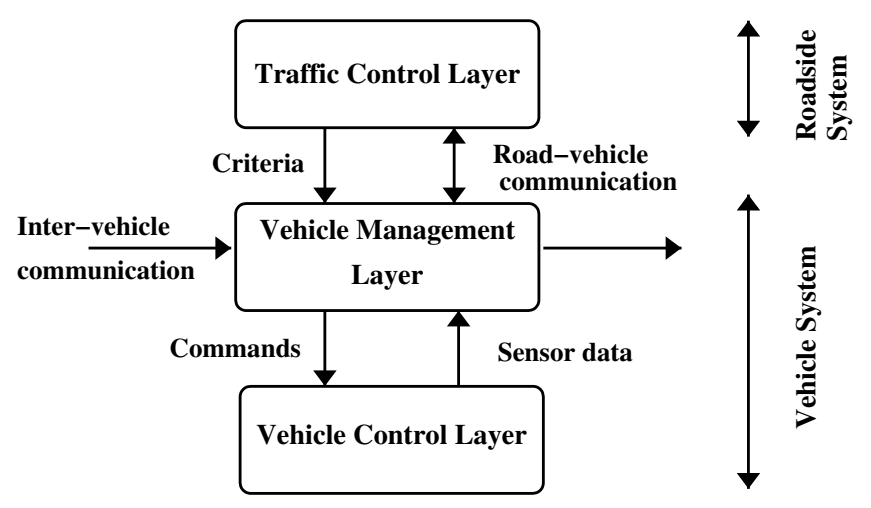

Figure 5: Dolphin architecture for cooperative driving

The vehicle management controller, which resides in each vehicle, receives suggestions for the movements of the vehicle from the traffic controller via road-vehicle communication and also considers the messages from the neighbouring vehicles via inter-vehicle communication and the data received from the basic vehicle control layer. This controller determines the movements of the individual vehicle under platoon-based driving. Lateral and longitudinal control actions for the platoon leader or free agent are determined by this layer using, e.g., a localisation function by GPS and a digital map of the traffic network. The vehicle management controllers in the follower vehicles determine both the lateral and the longitudinal control commands for the vehicle they control.

The traffic control layer is the top layer and it includes roadside IVHS equipment like sign boards, conventional lane markers, and variable message signs, as well as logic such as laws, rules, and common sense. There is only one traffic control layer common to all the vehicles and it is part of the roadside infrastructure. The traffic layer consists of several distributed controllers, each of which determines advisory instructions for the vehicles in its own neighbourhood and sends these instructions to the vehicle management layer. For example, the instructions could be to maintain a small inter-vehicle distance between the followers in a platoon, and to support an autonomous driving for the leaders in a platoon.

\subsection{Auto21 CDS framework}

The Auto21 Collaborative Driving System (CDS) framework [5] is mainly inspired by the concepts of the PATH and Dolphin architectures. The CDS architecture considers platoons of cars as autonomous agents and uses cooperative ACC technologies to support platoon-based driving. The CDS framework employs an inter-vehicle coordination system that can ensure coordination of vehicle activities during their merge and split operations from a platoon and that can maintain stability among the vehicles in a platoon. The hierarchical architecture of the Auto21 CDS framework consists of the following three layers as shown in Figure 6:

- Guidance layer,

- Management layer,

- Traffic control layer.

In every vehicle there is a guidance layer controller that senses the state of the vehicle and sends information on the position, speed, acceleration, etc. of the vehicle to the management layer. This 


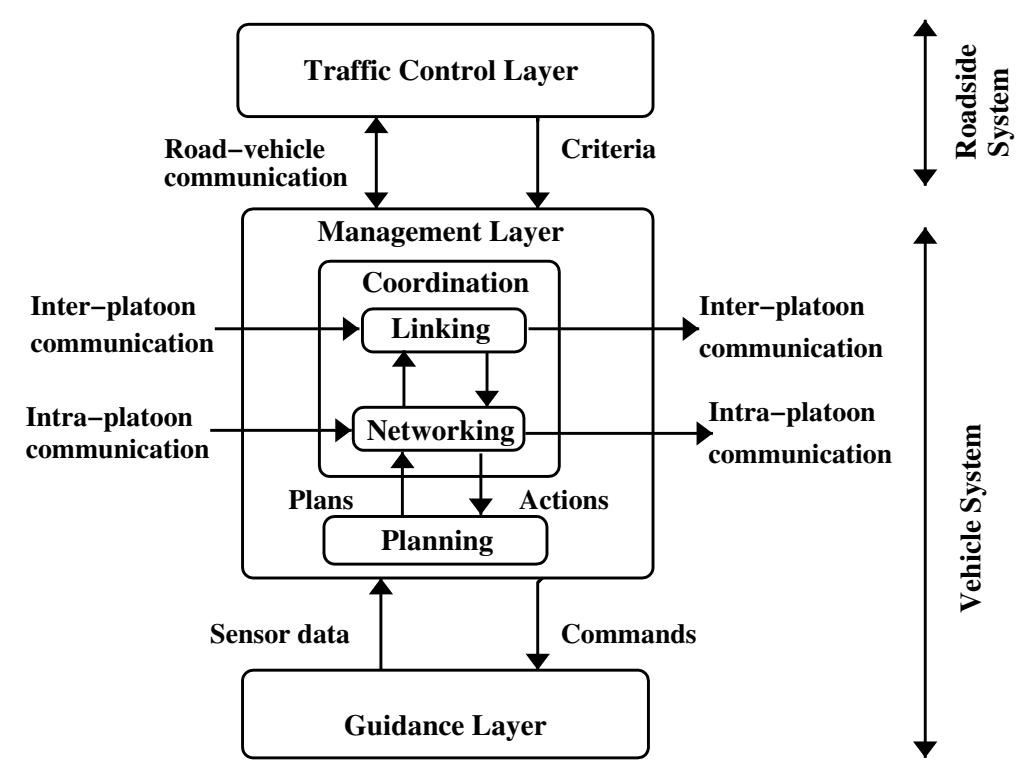

Figure 6: Auto21 CDS architecture for cooperative driving

controller also receives commands from the management layer, which are then translated into control actions for the throttle, braking, and steering.

The management layer is the main contribution of the CDS framework. The management layer contains several controllers, one for each vehicle. A management layer controller is responsible for determining the movements of the vehicle it manages using the information received from the traffic control layer, and makes sure that vehicle coordination constraints are satisfied through inter-vehicle communication. This layer is subdivided into coordination and planning sublayers that work cooperatively. The coordination sublayer has a linking module that communicates with the traffic control layer to receive suggestions for the lane change actions and that manages the inter-platoon activities. Once the linking module has made a choice on which the action to perform, the manoeuvres are executed (such as merge or split from a platoon) by a networking module, which coordinates the intra-platoon activities. The planning sublayer is responsible for making the plans to execute the manoeuvres inside the platoons. These plans are constructed in cooperation and coordination with the networking module.

The traffic control layer is located in the roadside infrastructure and provides suggestions on desired speeds for segments, maximum platoon sizes, etc. The traffic control layer also supports the linking module (of the management layer) for making decisions on inter-platoon coordination actions [38].

\subsection{CVIS}

CVIS (Cooperative Vehicle-Infrastructure Systems) [26,103] is a European research and development project that aims to design, develop, and test technologies that allow communication between the cars and with the roadside infrastructure, which in turn will improve road safety and efficiency, and reduce environmental impact. Traffic systems with CVIS technologies select a suitable communication medium depending on user requirements and available media, and allow cars to communicate in a secure way using wireless technologies.

CVIS operates with existing traffic control and management centres, roadside infrastructures, and 


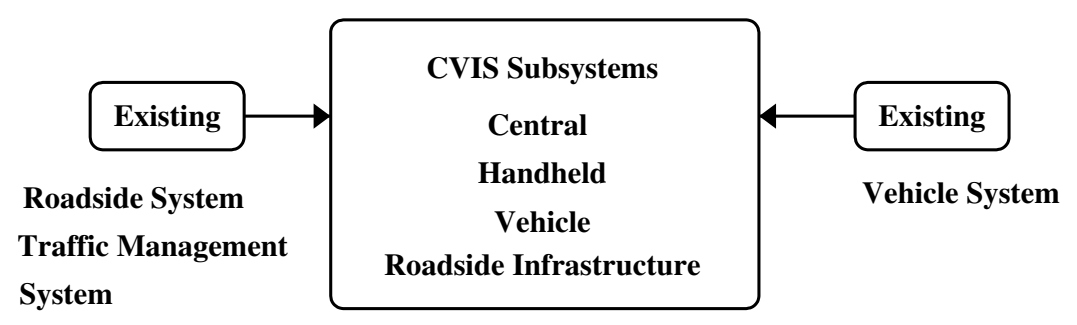

Figure 7: CVIS set-up

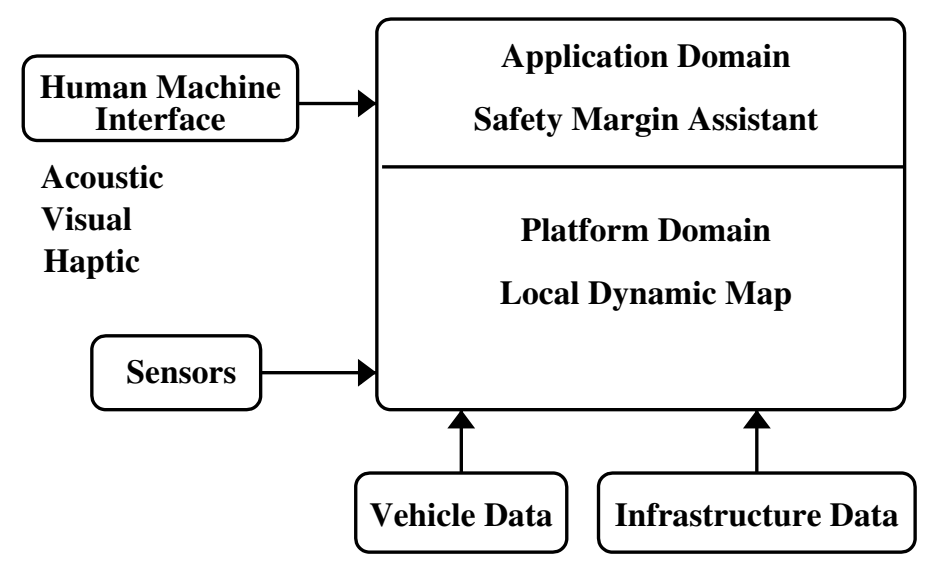

Figure 8: SafeSpot set-up

vehicle systems. The complete system can be considered as a single-level architecture with the existing systems and CVIS operating at the same level. Various networks and communication protocols have been developed within CVIS to enable communication between different subsystems. The time scale for this architecture ranges from minutes to hours.

A CVIS system is composed of four subsystems as shown in Figure 7: central, handheld, vehicle, and roadside subsystems. The central subsystem is a basically a service provider for the vehicle or the roadside infrastructure. Typical examples of central subsystems include traffic control and service centres, and authority databases. The handheld subsystem provides services such as pedestrian safety and remote management of other CVIS subsystems by allowing access to the CVIS system using PDAs and mobile phones. The vehicle subsystem is comprised of on-board systems and includes vehicle sensors and actuators, and equipment for vehicle-vehicle and vehicle-infrastructure communication. The roadside subsystem corresponds to the intelligent infrastructure that operates at the roadside and includes traffic signals, cameras, variable message signs, etc.

\subsection{SafeSpot}

SafeSpot $[90,100]$ is a research project funded by the European 6th Framework Program on Information Society Technologies. The main objective of this project is to improve road safety using advanced driving assistance systems and intelligent roads. The safety margin assistant developed by the SafeSpot project uses advanced communication technologies to obtain information about the surrounding vehicles and about the roadside infrastructure. This safety margin assistant can detect dangerous situations in advance and can make the driver aware of the surrounding environment using a human machine interface as shown in Figure 8. The time scale for this architecture ranges from 


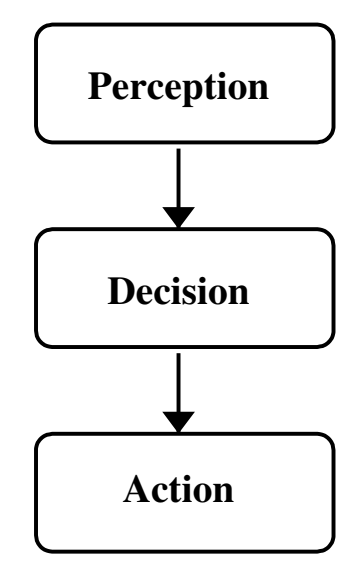

Figure 9: PReVENT set-up

seconds to minutes.

\subsection{PReVENT}

PReVENT [82] is a European automotive industry activity co-funded by the European Commission. The main focus of the PReVENT project is to develop preventive applications and technologies that can improve the road safety. These safety applications use in-vehicle systems to maintain safe speeds and distances depending on the nature and severity of the obstacles, and to provide instructions and to assist the drivers in their driving tasks so as to avoid collisions and accidents.

The PReVENT architecture introduces a three-layer approach as shown in Figure 9 with the following layers:

- Perception layer,

- Decision layer,

- Action layer.

All these layers are located within the vehicle. From the perception layer upward to the action layer, the time complexity and update frequency of states typically ranges from milliseconds to seconds.

The perception layer uses on-board sensors (such as radar, cameras, and GPS receivers) in conjunction with digital maps and allows vehicle-to-vehicle and vehicle-to-infrastructure communication.

The decision layer assesses dangerous situations ahead of the vehicle and determines relevant actions that are needed to avoid such situations. The controller then passes this decision to the action layer.

The action layer then issues warnings to the driver about the severity of the situation through an appropriate human machine interface or through vehicle actuators such as the steering wheel or the brakes.

\section{Comparison of the IVHS frameworks}

In Section 4 we have discussed various existing frameworks and architectures for IVHS-based traffic management. Now, we will compare the frameworks based on the following features: 
Table 2: Comparison of the various IVHS frameworks (in this table "veh" stands for "vehicles", "V2V" means "vehicle-vehicle", and "V2I" is short for "vehicle-infrastructure")

\begin{tabular}{|c|c|c|c|c|c|}
\hline Framework & $\begin{array}{c}\text { Control } \\
\text { objectives }\end{array}$ & $\begin{array}{c}\text { Formation } \\
\text { control }\end{array}$ & Intelligence & $\begin{array}{l}\text { Communication } \\
\& \text { coordination }\end{array}$ & $\begin{array}{l}\text { Time } \\
\text { scale }\end{array}$ \\
\hline PATH & $\begin{array}{l}\text { platoon manoeuvres } \\
\text { traffic flow efficiency }\end{array}$ & platoons & $\mathrm{road} / \mathrm{veh}$ & V2V/V2I & $\mathrm{ms}-\mathrm{h}$ \\
\hline Dolphin & platoon manoeuvres & platoons & $(\mathrm{road}) / \mathrm{veh}$ & $\mathrm{V} 2 \mathrm{~V}$ & $\mathrm{~ms}-\mathrm{h}$ \\
\hline Auto21 CDS & $\begin{array}{c}\text { intra-platoon } \\
\text { manoeuvres }\end{array}$ & platoons & $($ road $) /$ veh & $\mathrm{V} 2 \mathrm{~V}$ & $\mathrm{~ms}-\mathrm{h}$ \\
\hline CVIS & traffic efficiency & cars & $\mathrm{road} / \mathrm{veh}$ & $\mathrm{V} 2 \mathrm{~V} / \mathrm{V} 2 \mathrm{I}$ & $\min -\mathrm{h}$ \\
\hline SafeSpot & $\begin{array}{l}\text { prevent/avoid road } \\
\text { accidents }\end{array}$ & cars & (road)/veh & $\mathrm{V} 2 \mathrm{~V} / \mathrm{V} 2 \mathrm{I}$ & $\mathrm{s}-\mathrm{min}$ \\
\hline PReVENT & car safety & cars & veh & $\mathrm{V} 2 \mathrm{~V}$ & $\mathrm{~ms}-\mathrm{s}$ \\
\hline
\end{tabular}

- Control objectives,

- Type of formation control (platoons or single cars),

- Intelligence at roadside and/or in vehicles,

- Presence and type of communication and/or coordination,

- Time scales involved.

The results of this analysis are shown in Table 2.

\section{Control objectives}

For the PATH framework, the focus is primarily on designing and developing lateral and longitudinal controllers that allow automation of vehicles in a platoon and platoon manoeuvres. The PATH program has also developed coordination and communication techniques that are required during such manoeuvres. In addition, the roadside controllers in the PATH framework (i.e., the network and link layer controllers) determine the activities that need to be carried out in different freeway segments to avoid congestion. The Dolphin framework is vehicle-oriented, which means that the framework is mainly intended to study the inter-vehicle communication technologies that can be used to control a group of platoons that reside on neighbouring lanes and to support their manoeuvres. The AUTO21 CDS framework mainly aims at developing communication and coordination methodologies for merging and splitting actions within the platoon (intra-platoon manoeuvres). This framework uses an inter-platoon coordination model that was developed by the PATH project [6]. The objective of the CVIS project is to develop controllers and communication technologies for implementing a cooperative vehicle-highway system.

The PATH, Dolphin, AUTO21 CDS, and CVIS frameworks have developed control methodologies to be implemented in the roadside infrastructure to improve the traffic flow or in vehicles to allow automation of driving tasks. On the other hand, SafeSpot and PReVENT focus on improving the road safety by avoiding or preventing accidents, and they aim at integrated safety, with an emphasis on the potential of communication between vehicles and between vehicles and roadside systems. 


\section{Type of formation control}

The frameworks usually consider the vehicles to be controlled either as part of higher-level entities such as platoons, or as individual vehicles. The PATH, Dolphin, and Auto21 CDS frameworks allow platooning. On the other hand, SafeSpot, PReVENT, and CVIS do not use platoons.

\section{Intelligence at roadside and/or in vehicles}

The PATH framework allows involvement of both roadside infrastructure and vehicles for improving traffic performance. Although the Dolphin and the Auto21 CDS frameworks consider distributed intelligence between roadside infrastructure and vehicles, the roadside infrastructure only provides suggestions and instructions to the vehicles. The platoons are not obliged to follow these suggestions. CVIS and SafeSpot incorporate intelligence in both vehicle and roadside infrastructure. PReVENT also includes distributed intelligence but with the main focus on vehicle intelligence.

\section{Presence and type of communication and/or coordination}

Almost all the frameworks and projects have designed and developed technologies for inter-vehicle and roadside-vehicle communication for coordination of activities. Specifically, PATH has developed dedicated communication protocols $[35,50]$ and the Dolphin framework has developed a wireless local access network model for vehicle following, and inter-vehicle communication technologies for platoon manoeuvre coordinations [101]. For the coordination of tasks within the platoons, the AUTO21 CDS framework allows both a centralised set-up (i.e., the platoon leader instructs intraplatoon manoeuvres) or a decentralised set-up (i.e., all the members of the platoon are involved in the coordination)

SafeSpot, PReVENT, and CVIS focus on the issue of developing communication techniques that can be implemented in existing traffic networks and that can also be extended to AHS.

\section{Time scales involved}

The time scales involved for the PATH, Dolphin, and Auto21 CDS frameworks vary from milliseconds to hours as one traverses from dynamics of the vehicle up to the roadside infrastructure levels. The time complexity and update frequency typically ranges from minutes to hours for CVIS, from seconds to minutes for SafeSpot, and from milliseconds to seconds for PReVENT.

\section{Outlook}

In this section we first sketch how the control design methods presented in Section 2 could eventually be applied within the various IVHS control architectures of Section 4. We also indicate the challenges and issues that still have to be addressed to get a full-fledged and complete IVHS-based traffic management and control system.

\subsection{Application to IVHS}

In relation to IVHS, longitudinal controllers for IVs inside platoons such as ACC controllers can be implemented using static feedback control. In particular, we now discuss how the accelerations for the follower vehicles within a platoon could be calculated using a static feedback controller. The follower vehicles in a platoon should use their on-board ACC system to maintain short intra-platoon distances. 


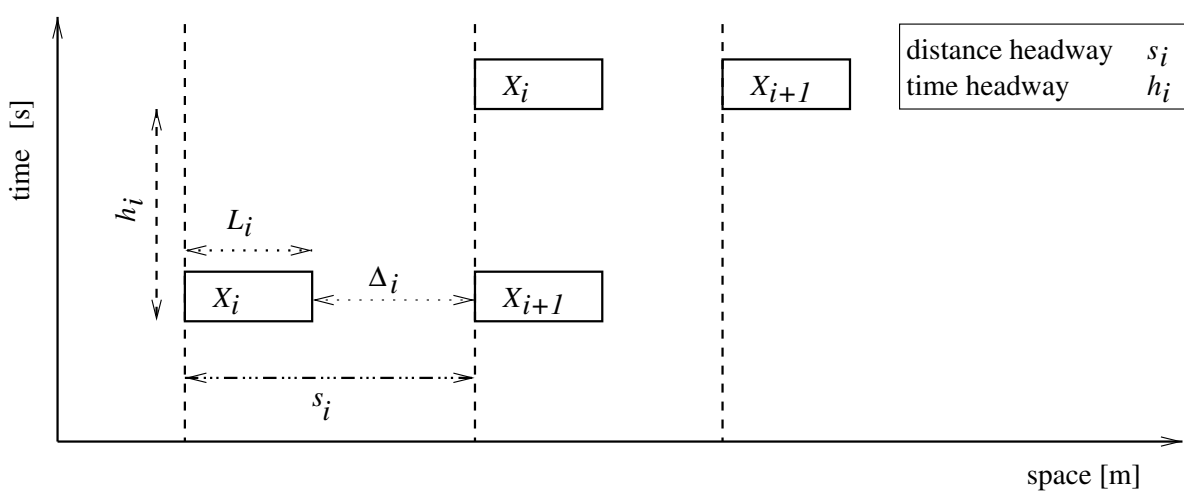

Figure 10: Time-space plot illustrating the definition of the time headway $h_{i}$ and the distance headway $s_{i}$ for vehicle $i$

Hence, an ACC algorithm could consist of a combined speed and distance controller as described in [51]:

$$
a_{i}(k)=K_{2}\left(h_{\text {ref }, i}(k)-\left(x_{i+1}(k)-x_{i}(k)\right)\right)+K_{3}\left(v_{i+1}(k)-v_{i}(k)\right)
$$

where $a_{i}(k)$ is the acceleration for vehicle $i$ at time $t=k T, v_{i}(k)$ is the speed of vehicle $i$ at time $t=k T$, $x_{i}(k)$ is the longitudinal position of the rear of vehicle $i$ at time $t=k T, h_{\text {ref }, i}$ is the reference distance headway for vehicle $i$, and $K_{2}$ and $K_{3}$ are controller parameters. These definitions are illustrated using two vehicles $i$ and $i+1$ travelling on a single lane in a time-space coordination system as shown in Figure 10. The time and distance directions are shown in vertical and horizontal axes respectively. The time headway is more often considered rather than distance headway because of its simplicity and ease of measuring. In practice, the distance headway can be obtained from photos.

Optimal control and in particular MPC can be applied either in vehicle controllers or in roadside controllers. For a low-level control tasks involving manoeuvres of individual vehicles such as ACC or ISA, which need frequent updates of the control signal (e.g., the acceleration), these methodologies may prove to be very slow due to the computational complexity involved. However, MPC is excellently suited for higher-level IV-based traffic control and management purposes and to determine optimal speeds, lane allocations, and on-ramp release times for the platoons. MPC offers a strategy that can determine the control actions based on the current and predicted future states of the traffic network, and that can optimise the performance objective assigned to roadside infrastructure over a given time horizon subject to the operational constraints and the constrained imposed on them. Moreover, the feedback and the receding horizon approach of MPC allow to reduce the effects of possible mismatch errors between the actual real-world traffic flows and the predicted traffic flows. Some results in this direction are described in [7,8]. In addition, since MPC is an optimisation-based approach, it is able to deal with the many different, often conflicting objectives (e.g., safety versus efficiency) that play a role in IVHS through the use of multi-criteria optimisation [71].

Fuzzy controllers can be used to implement an ACC controller using the deviations in speeds and distances as input variables. A typical example of such an application is dealt with in [46]. Moreover, fuzzy control can also be used to assist in making decisions for traffic management purposes. On the other hand, case-based reasoning will be more suitable for traffic planning and management purposes than for individual vehicle controllers. 


\subsection{Challenges and open issues}

Now we discuss the main technological, economical, and societal challenges that will have to be addressed when actually implementing an IVHS systems.

Although we have indicated above how control design methods such as static feedback control, MPC, and AI-based control could be used for IVHS and traffic management and control systems based on intelligent vehicles and platoons, real integration of these methods is still lacking. This is one of the challenges that still have to be addressed. However, the deployment of these control design methodologies does not ensure stability and robustness of the traffic system since IVHS and IVs are non-linear and often even hybrid (i.e., they exhibit both continuous dynamics and discrete-event behaviour (switching)).

The new control methods developed within PATH and the other frameworks have mainly focused on the lower layers, i.e., the coordination between the vehicles and the platoons, and have not really focused on higher-level traffic management aspects beyond the single freeway level such as route guidance and network-wide coordination. Moreover, the segment concepts used in PATH may lead to difficulties when dealing with very long platoons. Indeed, when the platoon size is very large, then a platoon might cover several segments, and then it might be difficult for the roadside controllers involved to assign the appropriate activities for the platoon, and also for the involved vehicle controllers to coordinate their activities. This raises two important open problems: platoon formation and scalability.

In literature there are no strict rules available on how to form platoons and on how many vehicles to include in a platoon. This can either be specific to a given road or to a destination. There are few articles that deal with vehicle sorting with respect to platoon sizes and platoon formation time, and also on the design of platoon manoeuvre protocols [37,49].

Some of IVHS frameworks such as the PATH, Auto21 CDS, and Dolphin frameworks are by nature hierarchical and offer thus a certain degree of scalability with regard to network size. Other frameworks such PReVENT, SafeSpot, and CVIS are not explicitly hierarchical and are thus not inherently scalable with regard to network size. However, none of the frameworks explicitly addresses scalability and the scalability of the frameworks has not yet been investigated in detail in literature. So this is also a topic for future research. In addition, scalability with regard to platoon sizes is also an open issue.

Hence, one of the major requirements is the development of a (new) integrated hierarchical traffic management framework for IVHS and the design and implementation of appropriate control methods for such a framework. Moreover, the full automation present in IVHS may also lead to new traffic control measures that can currently not be implemented, such as "real-valued" speed limits for more accurate control, smoother merging at on-ramps through speed and spacing control, etc. These new measures should also be accommodated in the control framework.

The technical issues outlined above are still open and need to be addressed. Moreover, the IVHS approach requires major investments to be made by both the government (or the body that manages the highway system) and the constructors and owners of the vehicles. Since few decisions are left to the driver and since the AHS assumes almost complete control over the vehicles, which drive at high speeds and at short distances from each other, a strong psychological resistance to this traffic congestion policy is to be expected. In addition, the fact that vehicles can be tracked through the entire network may raise some concerns regarding privacy and liability issues.

Another important question is how the transition of the current highway system to an AHS-based system should occur and - once it has been installed — what has to be done with vehicles that are not yet equipped for IVHS. As an intermediate step towards IVHS the current highway management 
system could start to communicate with the IVs and use information obtained from, e.g., the route guidance system of the IVs in order to more accurately forecast traffic loads. Other transition issues that have to be taken into account are [31]: How will the system be funded? What types of accidents can be expected to occur in AHS, in what numbers, and with what consequences? Will bi-directional communication and transfer of information be allowed between IVs and roadside infrastructure? What are the legal implications of an accident, especially if it were caused by system error or a system oversight? How will an AHS implementation be coordinated on an international level? etc.

\section{Summary}

We have presented on overview of traffic management and control frameworks for IVHS. First, we have given a short survey of the main control design methods currently used for freeway traffic control. Next, we have discussed various traffic management architectures for IVHS such as PATH, Dolphin, Auto21 CDS, CVIS, SafeSpot, and PReVENT. The frameworks have been compared in a qualitative way and we have sketched how the current traffic control methodologies could fit in an IVHS-based traffic control set-up. Finally, we have identified some open issues and future challenges in the further implementation and actual deployment of IVHS traffic management systems.

\section{Acknowledgements}

Research supported by the VIDI project "Multi-Agent Control of Large-Scale Hybrid Systems" (DWV.6188) of the Dutch Technology Foundation STW, Applied Science division of NWO and the Technology Programme of the Dutch Ministry of Economic Affairs, the BSIK projects "Transition to Sustainable Mobility (TRANSUMO)" and "Next Generation Infrastructures (NGI)", the European STREP project "Hierarchical and distributed model predictive control (HD-MPC)", the European COST Action TU0702, the Transport Research Centre Delft, and the Delft Research Centre Next Generation Infrastructures.

\section{References}

[1] A. Aamodt and E. Plaza. Case-based reasoning: Foundational issues, methodological variations, and system approaches. AI Communications, 7(1):39-59, March 1994.

[2] http://www.ahsra.or.jp/. Last visited on November 4, 2008.

[3] K. J. Åstrom, P. Albertos, M. Blanke, A. Isidori, W. Schaufelberger, and R. Sanz, editors. Control of Complex Systems. Springer-Verlag, New York, 2000.

[4] K. J. Åström and B. Wittenmark. Computer-Controlled Systems - Theory and Applications. PrenticeHall, Upper Saddle River, New Jersey, 3rd edition, 1997.

[5] http://www.damas.ift.ulaval.ca/projets/auto21/en/index.html. Last visited on December 2, 2008.

[6] S. Bana. Coordinating automated vehicles via communication. Technical Report UCB-ITS-PRR-200120, PATH Research Report, Berkely, California, 2001.

[7] L. D. Baskar, B. De Schutter, and J. Hellendoorn. Dynamic speed limits and on-ramp metering for IVHS using model predictive control. In Proceedings of the 11th International IEEE Conference on Intelligent Transportation Systems (ITSC 2008), pages 821-826, Beijing, China, October 2008.

[8] L. D. Baskar, B. De Schutter, and J. Hellendoorn. Model-based predictive traffic control for intelligent vehicles: Dynamic speed limits and dynamic lane allocation. In Proceedings of the 2008 IEEE Intelligent Vehicles Symposium (IV’08), pages 174-179, Eindhoven, The Netherlands, June 2008. 
[9] T. Bellemans, B. De Schutter, and B. De Moor. Model predictive control for ramp metering of motorway traffic: A case study. Control Engineering Practice, 14(7):757-767, July 2006.

[10] R. Bellman. Dynamic Programming. Princeton University Press, Princeton, New Jersey, 1957.

[11] R. Bishop. A survey of intelligent vehicle applications worldwide. In Proceedings of the IEEE Intelligent Vehicles Symposium, pages 25-30, Dearborn, Michigan, 2000.

[12] R. Bishop. http://www.ivsource.net/, 2005. Last visited on June 2, 2009.

[13] R. Bishop. Intelligent Vehicles Technology and Trends. Artech House, 2005.

[14] J. Blum and A. Eskandarian. Managing effectiveness and acceptability in intelligent speed adaptation systems. In International Conference on Intelligent Transportation Systems, pages 319-324, Toronto, Canada, September 2006.

[15] K. Bogenberger, H. Keller, and A. D. May. A neuro-fuzzy approach for ramp metering. In Proceedings of the 10th International Conference on Road Transport Information and Control, pages 101-105, London, England, 2000.

[16] M. Broucke and P. Varaiya. The automated highway system: A transportation technology for the 21st century. Control Engineering Practice, 5(11):1583-1590, November 1997.

[17] E. F. Camacho and C. Bordons. Model Predictive Control in the Process Industry. Springer-Verlag, Berlin, Germany, 1995.

[18] E. Camponogara, D. Jia, B. H. Krogh, and S. Talukdar. Distributed model predictive control. IEEE Control Systems Magazine, 22(1):44-52, February 2002.

[19] O. Carsten and F. Tate. Intelligent speed adaptation: The best collision avoidance system? In Proceedings of the 17th International Technological Conference on the Enhanced Safety of Vehicles, pages 1-10, Amsterdam, The Netherlands, 2001.

[20] C. G. Cassandras, D. L. Pepyne, and Y. Wardi. Optimal control of systems with time-driven and eventdriven dynamics. In Proceedings of the 37th IEEE Conference on Decision and Control, pages 7-12, Tampa, Florida, December 1998.

[21] H. Chen and F. Allgöwer. Quasi-infinite horizon nonlinear model predictive control scheme with guaranteed stability. Automatica, 34(10):1205-1217, January 1998.

[22] S. H. Chen, A. J. Jakeman, and J. P. Norton. Artificial intelligence techniques: An introduction to their use for modelling environmental systems. Mathematics and Computers in Simulation, 78(2):379-400, July 2008.

[23] D. W. Clarke, C. Mohtadi, and P. S. Tuffs. Generalized predictive control - Part I. The basic algorithm. Automatica, 23(2):137-148, March 1987.

[24] L. S. Comte. New systems: new behaviour? Transportation Research Part F, 3(2):95-111, May 2000.

[25] J. Cuena, J. Hernández, and M. Molina. Knowledge-based models for adaptive traffic management systems. Transportation Research Part C, 3(5):311-337, October 1995.

[26] http://www.cvisproject.org/. Last visited on November 4, 2008.

[27] C. F. Daganzo. Fundamentals of Transportation and Traffic Operations. Pergamon Press, 1997.

[28] S. Darbha and K. R. Rajagopal. Intelligent cruise control systems and traffic flow stability. Transportation Research Part C, 7(6):329-352, December 1999.

[29] L. C. Davis. Effect of adaptive cruise control systems on traffic flow. Physical Review E, 69:1-8, 2004.

[30] B. De Schutter, S. P. Hoogendoorn, H. Schuurman, and S. Stramigioli. A multi-agent case-based traffic control scenario evaluation system. In Proceedings of the IEEE 6th International Conference on Intelligent Transportation Systems (ITSC'03), pages 678-683, Shanghai, China, October 2003. 
[31] R. E. Fenton. IVHS/AHS: Driving into the future. IEEE Control Systems Magazine, 14(6):13-20, December 1994.

[32] A. V. Fursikov. Optimal Control of Distributed Systems: Theory and Applications. American Mathematical Society, Boston, Massachusetts, 2000.

[33] V. Gazi and K. M. Passino. Stability of a one-dimensional discrete-time asynchronous swarm. IEEE Transactions on Systems, Man and Cybernetics, Part B: Cybernetics, 35(4):834-841, August 2005.

[34] M. Gelfand and S. V. Fomin. Calculus of Variations. Dover Publications, New York, 1991.

[35] D. N. Godbole, J. Lygeros, E. Singh, A. Deshpande, and A. E. Lindsey. Design and verification of communication protocols for degraded modes of operation of AHS. In Proceedings of the 34th IEEE Conference on Decision and Control, pages 427-432, New Orleans, Louisiana, 1995.

[36] F. L. Hall and K. Agyemang-Duah. Freeway capacity drop and the definition of capacity. Transportation Research Record, (1320):91-98, 1991.

[37] R. Hall and C. Chin. Vehicle sorting for platoon formation: Impacts on highway entry and throughput. Transportation Research Part C, 13(5-6):405 - 420, 2005.

[38] S. Hallé, B. Chaib-draa, and J. Laumonier. Car platoons simulated as a multiagent system. In Proceedings of the 4th Workshop on Agent-Based Simulation, pages 57-63, Montpellier, France, 2003.

[39] D. Hammerstrom. Working with neural networks. IEEE Spectrum, 30(7):46-53, July 1993.

[40] F. Hayes-Roth. Rule-based systems. Communications of the ACM, 28(9):921-932, 1985.

[41] J. K. Hedrick, M. Tomizuka, and P. Varaiya. Control issues in automated highway systems. IEEE Control Systems Magazine, 14(6):21-32, December 1994.

[42] A. Hegyi, B. De Schutter, and J. Hellendoorn. Model predictive control for optimal coordination of ramp metering and variable speed limits. Transportation Research Part C, 13(3):185-209, June 2005.

[43] A. Hegyi, B. De Schutter, and J. Hellendoorn. Optimal coordination of variable speed limits to suppress shock waves. IEEE Transactions on Intelligent Transportation Systems, 6(1):102-112, March 2005.

[44] A. Hegyi, B. De Schutter, S. P. Hoogendoorn, R. Babuška, and H. van Zuylen. Fuzzy decision support system for traffic control centers. In Proceedings of the European Symposium on Intelligent Techniques (ESIT 2000), pages 389-395, Aachen, Germany, September 2000. Paper BC-01-2.

[45] M. R. Hestenes. Calculus of Variations and Optimal Control Theory. John Wiley and Sons, New York, 1966.

[46] R. Holve, P. Protzel, and K. Naab. Generating fuzzy rules for the acceleration control of an adaptive cruise control system. In Proceedings of the 1996 Biennial Conference of the North American Fuzzy Information Processing Society, pages 451-455, Berkeley, California, 1996.

[47] S. P. Hoogendoorn, B. De Schutter, and H. Schuurman. Decision support in dynamic traffic management. Real-time scenario evaluation. European Journal of Transport and Infrastructure Research, 3(1):21-38, 2003.

[48] R. Horowitz and P. Varaiya. Control design of an automated highway system. Proceedings of the IEEE: Special Issue on Hybrid Systems, 88(7):913-925, July 2000.

[49] A. Hsu, F. Eskafi, S. Sachs, and P. Varaiya. Design of platoon maneuver protocols for ivhs. Technical Report 96-21, California Partners for Advanced Transit and Highways PATH, University of California, Berkeley, California, 1991.

[50] A. Hsu, F. Eskafi, S. Sachs, and P. Variaya. Protocol design for an automated highway system. Discrete Event Dynamic Systems: Theory and Applications, 2(1):183-206, 1993.

[51] P. Ioannou and C. C. Chien. Autonomous intelligent cruise control. IEEE Transactions on Vehicular Technology, 42(4):657-672, November 1993. 
[52] N. R. Jennings. A roadmap of agent research and development. Autonomous Agents and Multi-Agent Systems, 1(1):7-38, July 1998.

[53] R. K. Jurgen. Smart cars and highways go global. IEEE Spectrum, 28(5):26-36, May 1991.

[54] P. Kachroo and K. Özbay. Feedback Control Theory for Dynamic Traffic Assignment. Advances in Industrial Control. Springer-Verlag, Berlin, 1999.

[55] A. Karimi and A. Gupta. Incident management system for Santa Monica Smart Corridor. In Proceedings of the 63rd Annual Meeting of the Institute of Transportation Engineers, pages 180-185, The Hague, The Netherlands, 1993.

[56] S. Kim, M. E. Lewis, and C. C. White III. Optimal vehicle routing with real-time traffic information. IEEE Transactions on Intelligent Transportation Systems, 6(2):178-188, June 2005.

[57] D. E. Kirk. Optimal Control Theory: An Introduction. Prentice-Hall, Englewood Cliffs, New Jersey, 1970.

[58] G. J. Klir and B. Yuan. Fuzzy Sets and Fuzzy Logic: Theory and Applications. Prentice-Hall, Upper Saddle River, New Jersey, 1995.

[59] A. Kotsialos and M. Papageorgiou. Freeway ramp metering: An overview. In Proceedings of the 3rd Annual IEEE Conference on Intelligent Transportation Systems (ITSC 2000), pages 228-239, Dearborn, Michigan, October 2000.

[60] A. Kotsialos and M. Papageorgiou. Efficiency and equity properties of freeway network-wide ramp metering with AMOC. Transportation Research Part C, 12(6):401-420, 2004.

[61] A. Kotsialos, M. Papageorgiou, C. Diakaki, Y. Pavlis, and F. Middelham. Traffic flow modeling of large-scale motorway networks using the macroscopic modeling tool METANET. IEEE Transactions on Intelligent Transportation Systems, 3(4):282-292, December 2002.

[62] A. Kotsialos, M. Papageorgiou, M. Mangeas, and H. Haj-Salem. Coordinated and integrated control of motorway networks via non-linear optimal control. Transportation Research Part C, 10(1):65-84, 2002.

[63] B. Krause and C. von Altrock. A complete fuzzy logic control approach for existing traffic control systems. In Mobility for Everyone, Proceedings of the 4th World Congress on Intelligent Transportation Systems, Berlin, Germany, October 1997. Paper no. 2045.

[64] N. E. Leonard and E. Fiorelli. Virtual leaders, artificial potentials and coordinated control of groups. In Proceedings of 40th IEEE Conference on Decision and Control, pages 2968-2973, Orlando, Florida, 2001.

[65] K. Li and P. Ioannou. Modeling of traffic flow of automated vehicles. IEEE Transactions on Intelligent Transportation Systems, 5(2):99-113, June 2004.

[66] Y. Liu, K. M. Passino, and M. M. Polycarpou. Stability analysis of one-dimensional asynchronous swarms. IEEE Transactions on Automatic Control, 48(10):1848-1854, October 2003.

[67] J. M. Maciejowski. Predictive Control with Constraints. Prentice-Hall, Harlow, England, 2002.

[68] A. D. May. Traffic Flow Fundamentals. Prentice-Hall, Englewood Cliffs, New Jersey, 1990.

[69] M. McDonald, N. B. Hounsell, and S. R. Njoze. Strategies for route guidance systems taking account of driver response. In Proceedings of the 6th Vehicle Navigation and Information Systems, pages 328 333, Seattle, Washington, July 1995.

[70] F. Middelham. Fuzzy logic demo. Technical report, Rijkswaterstaat, AVV, Rotterdam, The Netherlands, 1993. In Dutch.

[71] K. M. Miettinen. Nonlinear Multiobjective Optimization. Kluwer Academic Publishers, Boston, Massachusetts, 1999. 
[72] M. Molina, J. Hernández, and J. Cuena. A structure of problem-solving methods for real-time decision support in traffic control. International Journal of Human-Computer Studies, 49(4):577-600, October 1998.

[73] H. T. Nguyen and E. A. Walker. A First Course in Fuzzy Logic. Chapman \& Hall Press, Boca Raton, Florida, 2nd edition, 1999.

[74] S. A. Nsour, S. L. Cohen, J. E. Clark, and A. J. Santiago. Investigation of the impacts of ramp metering on traffic flow with and without diversion. Transportation Research Record, (1365):116-124, 1992.

[75] U. Palmquist. Intelligent cruise control and roadside information. IEEE Micro, 13(1):20-28, 1993.

[76] M. Papageorgiou. Applications of Automatic Control Concepts to Traffic Flow Modeling and Control. Lecture Notes in Control and Information Sciences. Springer-Verlag, Berlin, Germany, 1983.

[77] M. Papageorgiou, J. M. Blosseville, and H. Haj-Salem. Modelling and real-time control of traffic flow on the southern part of Boulevard Périphérique in Paris: Part II: Coordinated on-ramp metering. Transportation Research Part A, 24(5):361-370, September 1990.

[78] M. Papageorgiou, H. Hadj-Salem, and F. Middelham. ALINEA local ramp metering: Summary of field results. In Proceedings of the 76th Annual Meeting of the Transportation Research Board, Washington, DC, 1997. Paper no. 970032.

[79] P. M. Pardalos and M. G. C. Resende. Handbook of Applied Optimization. Oxford University Press, Oxford, England, 2002.

[80] L. E. Parker. Designing control laws for cooperative agent teams. In Proceedings of IEEE International Conference on Robotics and Automation, pages 582-587, Atlanta, Georgia, 1993.

[81] M. Persson, F. Botling, E. Hesslow, and R. Johansson. Stop and go controller for adaptive cruise control. In Proceedings of the IEEE International Conference on Control Applications, pages 1692-1697, Kohala Coast, Hawaii, 1999.

[82] http://www.prevent-ip.org/. Last visited on November 4, 2008.

[83] S. J. Qin and T. A. Badgwell. An overview of industrial model predictive control technology. American Institute of Chemical Engineers, 93(316):232-256, June 1997.

[84] B. S. Y. Rao and P. Varaiya. Roadside intelligence for flow control in an IVHS. Transportation Research Part C, 2(1):49-72, 1994.

[85] M. J. Richardson and D. Smith. Design of the driver interface for autonomous intelligent cruise control. In Proceedings of the IEE Colloquium on Design of the Driver Interface, pages 7/1-7/4, London, England, 1995.

[86] S. G. Ritchie. A knowledge-based decision support architecture for advanced traffic management. Transportation Research Part A, 24(1):27-37, January 1990.

[87] S. G. Ritchie and N. A. Prosser. Real-time expert system approach to freeway incident management. Transportation Research Record, (1320):7-16, 1991.

[88] D. Roseman and S. S. Tvedten. Santa Monica Freeway Smart Corridor project operational multi-agency traffic management and expert system. In Mobility for Everyone, Proceedings of the 4th World Congress on Intelligent Transport Systems, Berlin, Germany, October 1997. Paper no. 1032.

[89] S. Russell and P. Norvig. Artificial Intelligence: A Modern Approach. Prentice-Hall, Englewood Cliffs, New Jersey, 2003.

[90] http://www.safespot-eu.org/. Last visited on November 4, 2008.

[91] H. Shimizu, M. Kobayashi, and Y. Yonezawa. A route guidance system of a traffic network. In Proceedings of the 34th SICE Annual Conference, pages 1549-1552, Hokkaido, Japan, July 1995. 
[92] S. E. Shladover. PATH at 20 - History and major milestones. IEEE Transactions on Intelligent Transportation Systems, 8(4):584-592, December 2007.

[93] D. D. Šiljak. Decentralized Control of Complex Systems. Academic Press, San Diego, California, 1991.

[94] Y. J. Stephanedes, E. Kwon, and K. Chang. Control emulation method for evaluating and improving traffic-responsive ramp metering strategies. Transportation Research Record, (1360):42-45, 1992.

[95] J. M. Sussman. Intelligent vehicle highway systems: Challenge for the future. IEEE Micro, 1(1418):101-104, June 1993.

[96] H. J. Sussmann and J. C. Willems. 300 years of optimal control: From the brachystochrone to the maximum principle. IEEE Control Systems Magazine, 17(3):32-44, June 1997.

[97] R. S. Sutton and A. G. Barto. Reinforcement Learning: An Introduction. MIT Press, Cambridge, Massachusetts, 1998.

[98] H. Taale and F. Middelham. Ten years of ramp-metering in The Netherlands. In Proceedings of the 10th International Conference on Road Transport Information and Control, pages 106-110, London, England, April 2000.

[99] R. Thomas. Less is more. IEE Review, 49(5):40-43, May 2003.

[100] G. Toulminet, J. Boussuge, and C. Laurgeau. Comparative synthesis of the 3 main european projects dealing with cooperative systems (CVIS, SAFESPOT and COOPERS) and description of COOPERS demonstration site 4. In Proceedings of the 11th IEEE Conference on Intelligent Transportation Systems, pages 809-814, Beijing, China, 2008.

[101] S. Tsugawa, S. Kato, K. Tokuda, T. Matsui, and H. Fujii. An architecture for cooperative driving of automated vehicles. In Proceedings of the IEEE Intelligent Transportation Symposium, pages 422-427, Dearborn, Michigan, 2000.

[102] S. Tsugawa, S. Kato, K. Tokuda, T. Matsui, and H. Fujii. A cooperative driving system with automated vehicles and inter-vehicle communications in demo 2000. In Proceedings of the 2001IEEE Conference on Intelligent Transportation Systems, pages 918-923, Oakland, California, August 2001.

[103] B. van Arem. Cooperative vehicle-infrastructure systems: An intelligent way forward. Technical Report 2007-D-R0158/B, Verkeer en Vervoer, TNO, Delft, The Netherlands, 2007. In Dutch.

[104] B. van Arem, C. J. G. van Driel, and R. Visser. The impact of cooperative adaptive cruise control on traffic-flow characteristics. IEEE Transactions on Intelligent Transportation Systems, 7(4):429-436, 2006.

[105] P. Varaiya. Smart cars on smart roads: Problems of control. IEEE Transactions on Automatic Control, 38(2):195-207, February 1993.

[106] P. Varaiya and S. E. Shladover. Sketch of an IVHS systems architecture. In Vehicle Navigation and Information Systems, pages 909-922, Dearborn, Michigan, October 1991.

[107] S. Vukanovic and O. Ernhofer. Evaluation and field implementation of the fuzzy logic based ramp metering algorithm accezz. In Proceedings of the 9th IEEE Conference on Intelligent Transportation Systems, pages 437-441, Toronto, Canada, 2006.

[108] D. Watling and T. van Vuren. The modelling of dynamic route guidance systems. Transportation Research Part C, 1(2):159-182, June 1993.

[109] G. Weiss, editor. Multiagent Systems: A Modern Approach to Distributed Artificial Intelligence. MIT Press, Cambridge, Massachusetts, 1999.

[110] J. Weymann, J. L. Fargas, and J. J. Henry. Optimization of traffic dynamic route guidance with drivers' reactions in a queue-based model. IEEE Transactions on Systems, Man and Cybernetics, 25(7):11611165, July 1995. 
[111] X. Yao. Evolving artificial neural networks. Proceedings of the IEEE, 87(9):1423-1447, 1999.

[112] H. Zhang and S. G. Ritchie. Real-time decision-support system for freeway management and control. Journal of Computing in Civil Engineering, 8(1):35-51, 1994. 